\title{
Theoretical Investigation of Geometrical Effects of Blades on Mixing of Breathing Air in a Flow-Based Spirometer
}

\author{
Hamed Bagheri Mohammadreza Chimerad* Mahdi Sajjadi \\ AJA Radiation Sciences Research Center. Aja University of Medical Sciences Tehran, Iran
}

\begin{abstract}
According to the fact that, in comparison with the other spirometers, a flow-based spirometer benefits from a wide range of advantages such as being easy to use and calibrate, in this study, we make an attempt to design the optimum turbine just by changing the geometry of blades in order to increase the efficiency of a flow-based spirometer. In fact, the aim of the study is to design and simulate different types of blades in order to increase the mixing of breathing air and decrease resistance to breathing. To reach this aim, blades with different shear angles have been investigated and the most appropriate one has been reported.
\end{abstract}

Keywords: Spirometer, Flow-based, Turbine, Simulation

DOI: $10.7176 /$ APTA/80-01

Publication date:October $31^{\text {st }} 2019$

\section{Introduction}

Spirometer is an electronic or mechanical device routinely used to perform pulmonary function test to measure the amount and speed of air that a person can inhale or exhale. Pulmonary function tests (PFTS) are invaluable clinical investigations performed with the purpose of diagnosis, monitoring the treatment and guiding decisions regarding further treatment and intervention for the patients with suspected or previously diagnosed respiratory disease.(Ranu et al. 2011)

As the most common procedure in performing pulmonary function tests, Spirometry is the process of obtaining parameters relating to lungs and respiratory function by means of measuring flow and/or volume of the exhaled and inhaled air. Inspired and expired lung volumes measured by spirometry are useful for detecting, characterizing and quantifying the severity of lung disease.(Quajer et al. 2011)

The medical doctor may use these data for diagnosis, treatment or monitoring therapy process or abnormalities developments for a wide variety of respiratory system related diseases.(Rebuck et al. 1996)

The device used in this process is called Spirometer. Spirometer devices are divided into two categories with respect to their measuring method, volume-based and flow-based spirometers. Volume-based spirometer are less expensive and yet easier to calibrate and use, but they are incapable of measuring flow parameters of pulmonary test, such as Peak Expiratory Flow(PEF).(Wanger et al. 2005)

Nowadays, digital flow-based spirometers are more common due to their precision, portability and the capability of measuring both flow and volume parameters for PFT. There are different types of flow-based spirometers that use various techniques in measuring the respiratory flow such as ultrasonic sensor, hot wire anemometer, venture sensor and turbine. Turbine spirometers are the most common types due to their ease of operation, adequate precision, light weight, cost efficiency and the capability to remove and change parts in contact with breathing gasses of patients to prevent disease transmission.(Rebuck et al. 1996)

Solving spirometry problems requires spirometers to meet a lot of conditions for performing their function adequately. These conditions have been developed over many years and defined now as medical/ technical requirements (MTR) for main features of devices. One of the main features to achieve a desirable precision of measurements in designing flow-based and in particular turbine spirometers is the increase of the mixing air through the blades of turbine. Furthermore, another importance of geometric design of blades which can have a significant effect on the process of measuring lung volumes is to reach the minimum resistance to breathing, created by spirometer. The negligible error in each mentioned parameter can be resulted in a significant fault in different criteria such as residual volume (RV), functional residual capacity (FRC) and total lung capacity (TLC). Consequently, the design of appropriate blades for the turbine of flow-based spirometer stands out as the substantial challenge.(Wanger et al. 2005)

In present study various types of blades have been designed and the effect of geometrical features of blades on the air mixing and resistance to breathing has been evaluated. Finally, based on the final results, an appropriate blade for the turbine design has been suggested.

\section{Materials and methods}

In this study, the flow-based spirometer has been investigated. Therefore, the cylindrical structure should be designed to indicate the process of spirometry. First, we make an attempt to scheme the basic structure of the flowbased turbine with the help of CATIA (computer-aided three-dimensional interactive application) software which has been shown in figure 1. According to figure 1, three different ingredients form the oral part of spirometer 
which can be mentioned as the initial part, fixed and moving blades. Due to the nature of fixed blades, breathing air moves through the fixed blades being located in the internal part of the spirometer and before facing the moving blades it transforms from irrotational into a rotational flow which can result in the movement of moving blades. To be more specific, breathing air flows through the turbine cylinder causing the propeller (moving blades) to spin. Secondly, we design various types of blades with changing the quantity and geometry of propellers. Finally, we make an effort in order to simulate the movement of breathing air through fixed blades with the aim to predict the optimum number and geometry of blades providing the minimum proportion of resistance to breathing into the rotational speed of the air. In the following sections, we will discuss the process of design and simulation of blades.

\subsection{Blades design}

Based on the medical/ technical requirements (MTR), one of the main features of spirometry devices is to achieve the least resistance to breathing air. Hence, designing the appropriate model for the blades and turbine of flowbased spirometer attaches importance to the device function. To achieve the optimum turbine and blades scheme, we design the basic model of them just by following the commercial patterns such as Mir reusable turbine. In next step, we design turbines just by changing their profiles. To shed more light on the issue, different types of profiles have various number of variables such as bending and shear angles, impeller diameter and form of baffles. In our study, for the purpose of minimizing the computation time, we restrict the variables into two distinctive parameters which are as follows: shear angles and impeller diameter. The quantity of the variables is indicated in Table1. According to them, our schemes have been limited into five different profiles. Table 2

In addition to geometrical variables of blades' profile, we examine a wide range of blade numbers from three to six in order to fulfill our goals. Table 1

\subsection{Blades simulation}

In practice, our design does not have adequate capability in order to conduct us to the appropriate result without carrying-out a computer simulation of breathing air movement through fixed blades. With the aim to simulate the airflow, we benefit from COMSOL Multiphysics software providing an interactive environment for modeling and simulating the breathing air movement. We run a wide range of simulation models to predict the optimum design for the spirometer turbine. In fact, we custom air as a fluid flowing through the blades with the fluid density of 1000. In addition, we use various quantities of shear angles and impeller diameter based on Table $1 \mathrm{a}, \mathrm{b}$ in order to satisfy our final goal. Furthermore, according to the software library, we use a variety of elements for the mesh generation step such as Tetrahedral, Pyramid, Prism, Triangular and Quadrilateral elements to reach an appropriate precision of the simulation.

\subsection{Drag equation and Eddy diffusivity}

After designing and simulating the turbine with fixed blades, we must clearly understand an appropriate scheme for the spirometer turbine. Therefore, we classify our results into three different categories based on our variables. In fact, we provide a number of figures in order to make a precise comparison between variables. To investigate their effects, we introduce Eddy diffusion and drag force as the parameters explaining the breathing air mixing and resistance to breathing, respectively. In fact, according to the data achieving from computer simulation, we analyze the geometrical impact of blades on the parameters helping us to reach an appropriate design for the flow-based turbine.

In fluid dynamics, the drag equation is a formula used to calculate the force of drag experienced by an object due to movement through a fully enclosing fluid. .(Y.Niknafs et al. 2011) The equation is defined by mass density, flow velocity, area, drag coefficient (a dimensionless coefficient related to the object's geometry and taking into account both skin friction and form drag. In general, it depends on the Reynolds number. The equation can be represented as:

$$
F_{D}=\frac{1}{2} \rho u^{2} C_{D}
$$

In fluid dynamics, Eddy diffusion is any diffusion process by which substances are mixed in the atmosphere or in any fluid system due to eddy motion. (I.P.Alves et al. 2012)

Because the responsible microscopic processes for atmospheric mixing are too complex to be modelled in detail, atmospheric modelers generally treat atmospheric mixing as a macroscopic Eddy diffusion process. In this approach, the diffusion rate at each pressure level is parameterized by a quantity known as the eddy diffusion coefficient with the unit of $m^{2} / s$.

\section{Results and Discussion}

First of all, in case of blades variation, the raw data showed increasing quantity of blades leads to a substantial rise in the value of Eddy diffusion and drag force which directly justify the increase in breathing air mixing and resistance to breathing. In addition, it seems obvious after the rapid increase in Eddy diffusion and drag force, they 
remain steady approximately. Figure 1

Furthermore, according to the results of Figure 2, a, with the rise in shear angles from 30 to 60 degree, there is a dramatic increase in the value of Eddy diffusion. This trend changes when shear angles vary from 60 to 85 , though. In fact, the Eddy Diffusion become leveled off and it remains constant. In addition, with the improvement in the value of shear angles, drag force fluctuates from 80 to 70 Newton and it reaches the lowest point when the shear angle is 60 degrees.

After that, the results of Table 3 show that the impeller diameter variation does not have significant effects on Eddy diffusion and Drag force approximately. In fact, with the variation of Impeller diameter from 1 to $2.6 \mathrm{~mm}$ the Eddy diffusion and Drag force varies from 40- $50 \mathrm{~m}^{\wedge} 2 / \mathrm{s}$ and $68-72 \mathrm{~N}$, respectively.

\section{Conclusion}

Consequently, based on the computational results, first of all, changing Impeller diameter does not have substantial impact on the breathing air mixing and resistance to breathing. However, the alteration of shear angles and blades numbers can have a dramatic effect on the mentioned parameters.

After that, based on the survey, in order to reach the maximum efficiency in the spirometer, we should have the maximum value Eddy diffusion and the minimum quantity of drag force. In other words, they enable us to reach the maximum quantity of air breathing mixing and the minimum value of resistance to breathing. According to figure 4, the best quantity of blades is 6 and the best shear angle is 60 degrees.

\section{References}

Ranu, H., Wilde, M., \& Madden, B. (2011). Pulmonary function tests. The Ulster medical journal, 80(2), 84.

Quanjer, P. H., Tammeling, G. J., Cotes, J. E., Pedersen, O. F., Peslin, R., \& Yernault, J. C. (1993). Lung volumes and forced ventilatory flows.

Rebuck, D. A., Hanania, N. A., D'Urzo, A. D., \& Chapman, K. R. (1996). The accuracy of a handheld portable spirometer. Chest, 109(1), 152-157.

Wanger, J., Clausen, J. L., Coates, A., Pedersen, O. F., Brusasco, V., Burgos, F., ... \& Gustafsson, P. (2005). Standardisation of the measurement of lung volumes. European respiratory journal, 26(3), 511-522.

DePold, H. R., \& Gass, F. D. (1998, June). The application of expert systems and neural networks to gas turbine prognostics and diagnostics. In ASME 1998 international gas turbine and aeroengine congress and exhibition (pp. V005T15A009-V005T15A009). American Society of Mechanical Engineers.

Schroeder, W., Martin, K., and Lorensen, B. (1998). The Visualization Toolkit. Prentice-Hall: New Jersey, 432435.

Litwin, L. (2000). FIR and IIR digital filters. IEEE potentials, 19(4), 28-31.

Yin, L., Yang, R., Gabbouj, M., \& Neuvo, Y. (1996). Weighted median filters: a tutorial. IEEE Transactions on Circuits and Systems II: Analog and Digital Signal Processing, 43(3), 157-192.

Y.Niknafs Abrebekooh, M.Rad (2011). Experimental and numerical investigation of drag force over tubular frustum. Scientia Irania, Pages 1133-1137.

I.P.Alves, G.A. Degrazia, D.Buske, M.T.Vihena, O.L.L. Moraes, O.C. Acevedo (2012) Derivation of an eddy diffusivity coefficient depending on source distance for a shear dominated plantary boundry layer. Physica A: Statistical Mechnaics and its Applications. Pages 6577-6586 

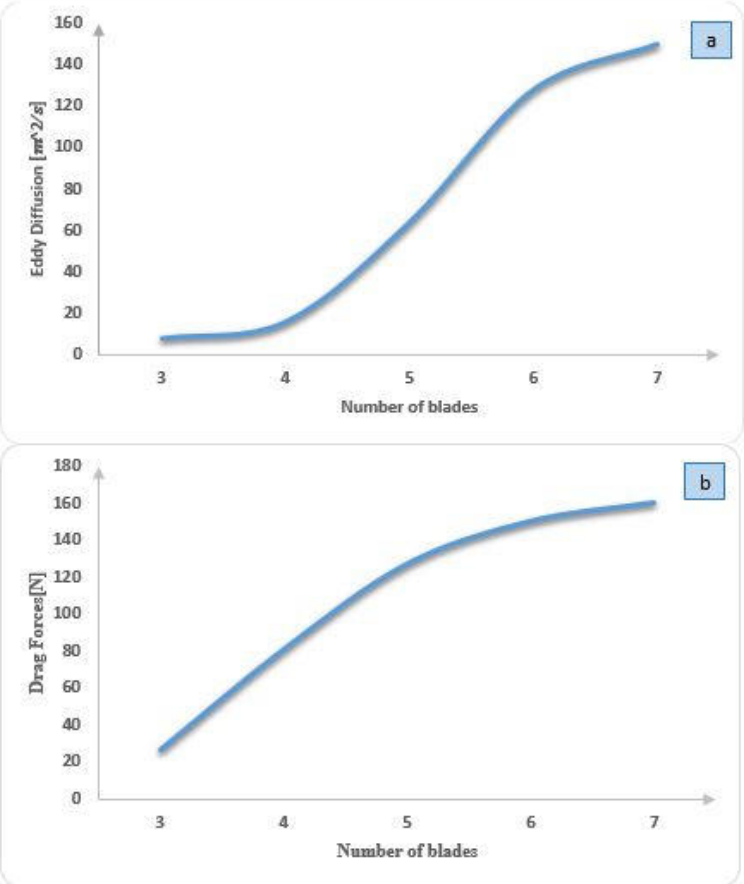

Figure 1. Effect of number of blades on drag force and Eddy diffusion
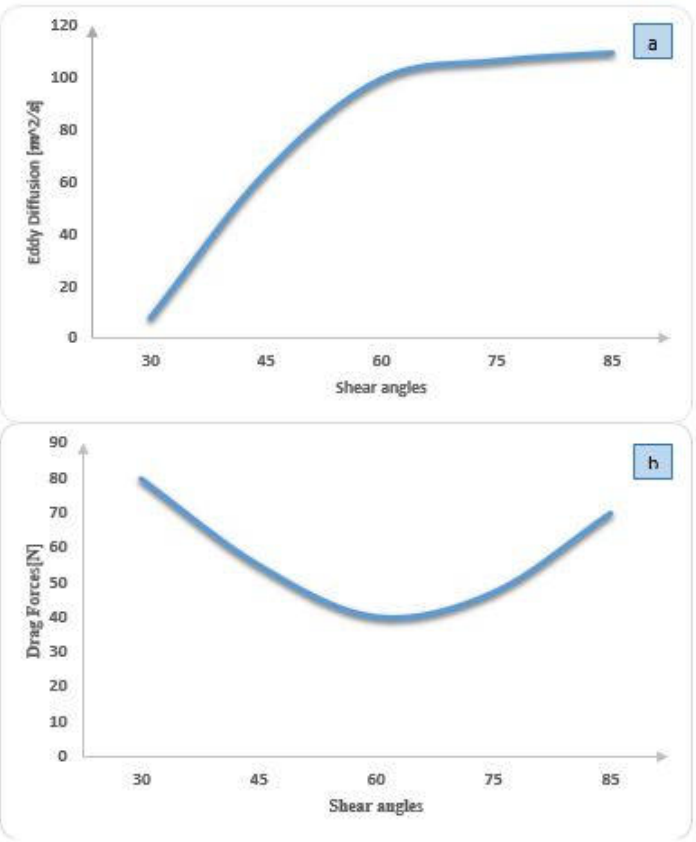

Figure 2. Effect of Shear angle on drag force and Eddy diffusion 

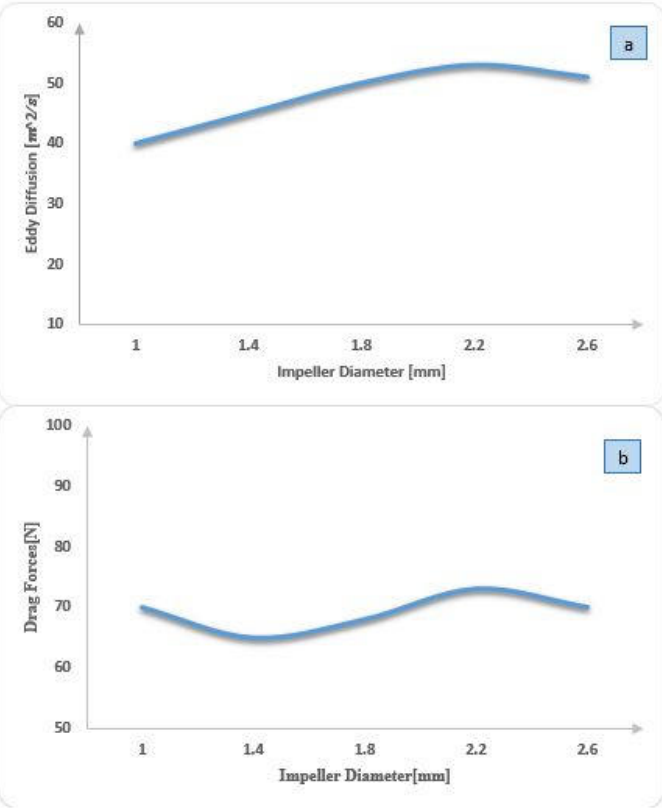

Figure 3. Effect of Impeller diameter on drag force and Eddy diffusion

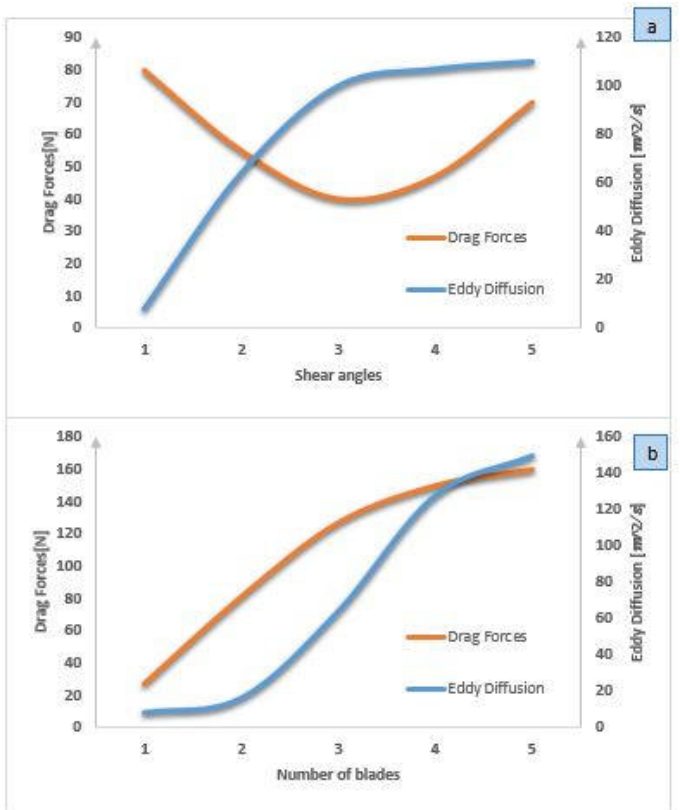

Figure 4. Effect of shear and number of blades on Drag force and Eddy diffusion

Table 1. The spirometer turbine variables being examined in the computational simulation.

\begin{tabular}{|l|c|c|c|c|c|}
\hline $\begin{array}{l}\text { Spirometer turbine } \\
\text { variables }\end{array}$ & \multicolumn{5}{|c|}{ The quantity of variables } \\
\hline Shear angles & $30^{\circ}$ & 45 & 60 & 75 & 85 \\
\hline Impeller diameter & $1^{*}$ & 1.4 & 1.8 & 2.2 & 2.6 \\
\hline Number of blades & 3 & 4 & 5 & 6 & 7 \\
\hline \multicolumn{7}{|c|}{$\begin{array}{l}\text { o. Degree unit } \\
\text { * Length unit (Millimeter) }\end{array}$}
\end{tabular}


Table 2. The samples of turbine being designed based on geometry and number of blades variation

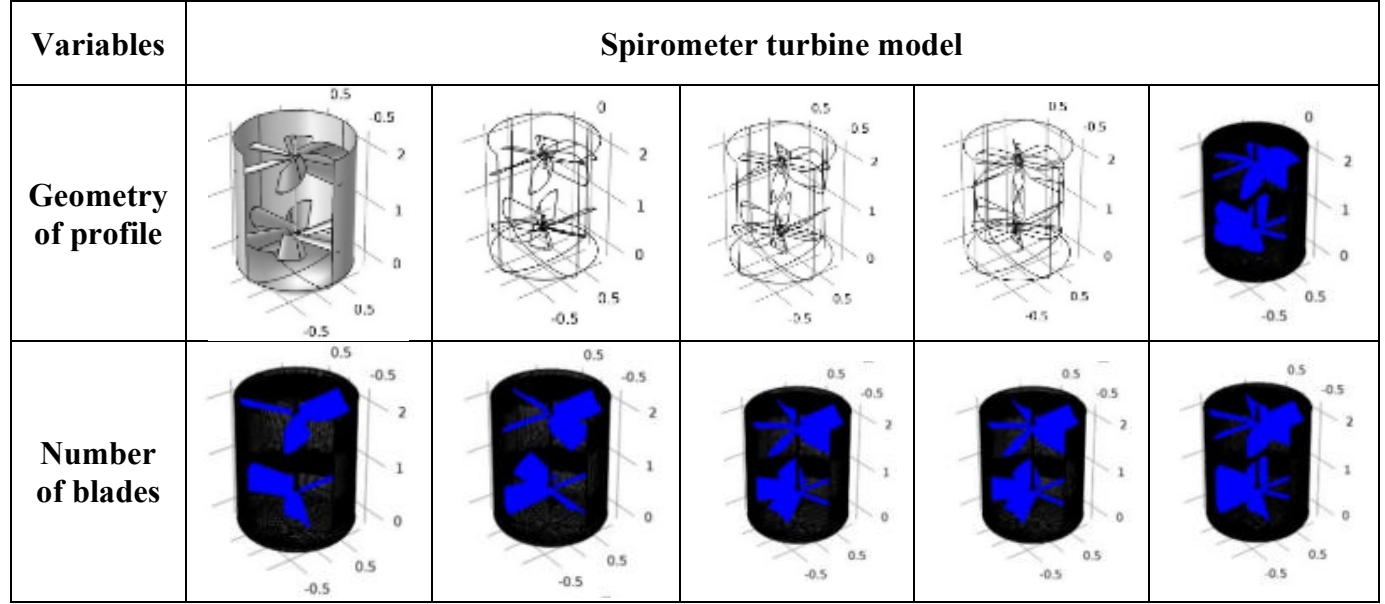

Table 3. The description of numbers locating in the horizontal axis in the figure 4

\begin{tabular}{|l|c|c|c|c|c|}
\hline \multicolumn{7}{|c|}{ Figure 4 description } \\
\hline $\begin{array}{l}\text { The mentioned } \\
\text { numbers }\end{array}$ & 1 & 2 & 3 & 4 & 5 \\
\hline Shear angles & 30 & 45 & 60 & 75 & 85 \\
\hline Number of blades & 3 & 4 & 5 & 6 & 7 \\
\hline
\end{tabular}

